\title{
An interesting case of preseptal cellulitis
}

\author{
Eddie Liu (Meds 2014) \\ Faculty Reviewer: Dr Saira Zafar, MD, FRCPC (Department of Medicine, Division of General Internal Medicine)
}

\section{CASE PRESENTATION}

A 39-year-old woman presents to the Emergency Department with swelling and erythema of her face involving the right eye. Her symptoms were preceded by a 6-day history of sore throat, fever and chills. Her medical history is significant for depression and she is on sertraline $25 \mathrm{mg}$ P.O. daily. On exam the patient had diffuse erythema and swelling in the lower half of her forehead and the entire region surrounding her right eye. Her right eye was swollen shut. Gross examination of the eye revealed no proptosis, no ophthalmoplegia and normal ocular movements. Pupils were round and equal with normal direct and consensual response. A CT scan of the head showed no involvement of the orbit and sinuses. Blood culture, urine culture and antistreptolysin O titre (ASOT) were all negative. Interestingly, the patient's husband was admitted to hospital a few days earlier with aggressive leg cellulitis and her child recently had strep throat and impetigo. Although no infectious organism was identified in this patient, Streptococcus pyogenes was isolated in an area of cellulitis on her husband's leg. For this reason, she was treated for S. pyogenes with ceftriaxone $1 \mathrm{mg}$ IV OD for three days followed by cefuroxime $500 \mathrm{mg}$ P.O. BID for 10 days.

\section{DISCUSSION}

It is important to distinguish between preseptal cellulitis and orbital cellulitis. The two conditions share similar clinical symptoms of ocular pain and eyelid swelling and erythema, but they have drastically differently clinical implications. ${ }^{1}$ Preseptal cellulitis, also known as periorbital cellulitis, is an infection of the anterior portion of the eyelid. It is generally a mild condition that rarely leads to serious complications. ${ }^{5}$ Conversely, orbital cellulitis is an infection that involves the contents of the orbit, such as the ocular muscles and fat, but not the globe. ${ }^{2}$ Orbital cellulitis can cause loss of vision and can be fatal. Clinical features that indicate orbital cellulitis include ophthalmoplegia, pain with eye movement and proptosis. ${ }^{7}$ In addition, imaging studies such as CT scans of the head can help to distinguish between the two. ${ }^{1}$ Even though preseptal cellulitis is more common than orbital cellulitis, in cases where distinction is unclear, patients should be treated as though they have orbital cellulitis due to the serious complications associated with untreated orbital cellulitis. In the case above, the patient exhibited clinical symptoms indicative of preseptal cellulitis and CT head ruled out any orbital involvement.

The treatment for preseptal cellulitis is usually empirical and depends on knowledge of the common infecting organisms and their antibiotic susceptibility. ${ }^{3}$ The most common organisms include Staphylococcus aureus, Streptococcus pneumoniae, other streptococci and anaerobes. If orbital cellulitis is suspected, a referral to an ophthalmologist should be made for further investigations. ${ }^{6}$ In this case the infectious organism was likely to be S. pyogenes because two of the patient's family members had recent streptococcal infections: cellulitis, strep throat and impetigo. It is interesting that her ASOT was tested negative; however the test is associated with a false negative rate of $20 \%-30 \%$ and does not definitively rule out a streptococcal infection on its own. ${ }^{4}$

\section{SUMMARY}

Preseptal and orbital cellulitis are two conditions that share similar clinical symptoms of swelling, erythema and ocular pain. Although preseptal cellulitis is more common and generally a mild condition, it is important to distinguish it from orbital cellulitis, which can lead to loss of vision and death. A careful history and physical can be extremely helpful in distinguishing the two conditions and may lead to identification of the offending organism. CT scans of the head are often advisable to rule out orbital and sinus involvement. Preseptal cellulitis is a condition that can be managed by antibiotics as an outpatient. If orbital cellulitis is suspected, however, an ophthalmology referral is warranted and hospital admission is strongly recommended.

\section{REFERENCES}

1. Botting AM, McIntosh D, Mahadevan M. Paediatric pre- and post-septal peri-orbital infections are different diseases. A retrospective review of 262 cases. Int J Pediatr Otorhinolaryngol. 2008 Mar;72(3):377-83.

2. Hofbauer JD, Gordon LK, Palmer J. Acute orbital cellulitis after peribulbar injection. Am J Ophthalmol. 1994 Sep;118(3):391-2.

3. Howe L, Jones NS. Guidelines for the management of periorbital cellulitis/abscess. Clin Otolaryngol Allied Sci. 2004 Dec;29(6):725-8.

4. Hahn RG, Knox LM, Forman TA. Evaluation of poststreptococcal illness. Am Fam Physician. 2005 May;71(10):1949-54.

5. Smith TF, O'Day D, Wright PF. Clinical implications of preseptal (periorbital) cellulitis in childhood. Pediatrics. 1978 Dec;62(6):1006-9.

6. Uzcátegui N, Warman R, Smith A, Howard CW. Clinical practice guidelines for the management of orbital cellulitis. $\mathrm{J}$ Pediatr Ophthalmol Strabismus. 1998 Mar-Apr;35(2):73-9.

7. Varma D, Metcalfe TW. Orbital cellulitis after peribulbar anaesthesia for cataract surgery. Eye (Lond). 2003 Jan;17(1):105-6. 